\title{
Older Patients' Perspectives of Online Health Approaches in Chronic Obstructive Pulmonary Disease
}

Rebecca T. Disler, $P h D^{1}{ }^{1}$ Sally C. Inglis, $P h D_{,}{ }^{2}$ Phillip Newton, $P h D_{1}^{2, *}$ David C. Currow, $P h D_{1}^{2}$

Peter S. Macdonald, $P h D_{1}^{3}$ Allan R. Glanville, $P h D_{1}^{3}$ Doranne Donesky, PhD, ${ }^{4}$ Virginia Carrieri-Kohlman, $P h D,{ }^{4}$ and Patricia Mary Davidson, $P h D^{2,5}$

\footnotetext{
${ }^{1}$ Department of Rural Health, The University of Melbourne, Shepparton, Australia.

${ }^{2}$ Centre for Improving Palliative, Aged and Chronic Care through Clinical Research and Translation, Faculty of Health, University of Technology Sydney, Broadway, Australia.

${ }^{3}$ Department of Thoracic Medicine, St. Vincent's Hospital, Sydney, Australia.

${ }^{4}$ Department of Physiological Nursing, University of California San Francisco School of Nursing, San Francisco, California.

${ }^{5}$ School of Nursing, Johns Hopkins University, Baltimore, Maryland.

${ }^{*}$ Current affiliation: School of Nursing and Midwifery, Western Sydney University, Sydney, Australia.
}

\begin{abstract}
Background: Chronic obstructive pulmonary disease (COPD) is a complex, chronic condition. Patients commonly have limited access to face-to-face support due to decreased mobility, symptom burden, and availability of services. Online health care approaches provide the potential for increased access to self-management education and support. This study sought to understand older patients with COPD's perspectives of online approaches to health care.

Materials and Methods: Participants older than 65 years were recruited from a respiratory service at an academic medical center. Qualitative, focus groups were used and recorded, transcribed verbatim, and analyzed using thematic analysis to identify key and repeated emergent themes.

Results: Focus groups were undertaken between January and May 2014. Thematic analysis resulted in five overall themes: (1) concern over risks in the online environment; (2) multimedia and technology use as part of everyday life; (3) online resources as an opportunity for revision of forgotten knowledge; (5) potential for facilitation of decision-making support across geographical and physical barriers; and (4) perceived benefits of online peer support for people with COPD.
\end{abstract}

Conclusions: Overall, these older participants with COPD had positive views of online health information, but did raise the need for guidance to ensure valid and reliable online sources. The capacity for online sources to increase access to decision support and up-to-date information was viewed positively, as was the ability to interact with peers who had similar experiences. Telecommunication tools and approaches are already being utilized in health care interactions. Further research is required into the most appropriate, feasible, and sustainable online health approaches to support patients with chronic illnesses such as COPD.

Keywords: chronic obstructive pulmonary disease, self-care, telehealth care, e-health, qualitative, telemedicine

\section{Introduction}

he increasing burden of noncommunicable diseases, such as heart and respiratory disease, is placing growing pressure on health systems globally. ${ }^{1,2}$ Chronic obstructive pulmonary disease (COPD) is a leading cause of morbidity and mortality, ranked fourth cause of death worldwide. ${ }^{1,2}$ With significant economic burden, COPD accounts for $\sim 6 \%$ of total health care budget in the European Union ( $€ 38.6$ billion). ${ }^{1,2}$ Social costs include significant disability due to daily symptoms, poor physical functioning, social isolation, and caregiver burden. ${ }^{3}$

Patients with COPD often struggle to access face-to-face services due to decreased mobility and symptom burden. ${ }^{3}$ Pulmonary rehabilitation, for example, is recommended internationally for self-management education in COPD; however, this primarily face-to-face approach has limited uptake and completion rates, with major barriers due to geography, transport, and logistical issues. ${ }^{2,4,5}$ Self-management education is defined in this context as training "to help patients acquire and practice the skills they need to carry out disease-specific medical regimens, to guide changes in health behavior, and to provide emotional support to enable patients to adjust their roles for optimal function and control of their disease."

Issues of health care access, increasing health costs, and the need for improved health outcomes drive the search for alternate and sustainable approaches to chronic disease management. ${ }^{2,3,7,8}$ Technology platforms may provide opportunities to support 
larger populations and for those outside the reach of facilitybased services in particular. ${ }^{4,5,8}$ While technology use is pervasive in society, knowledge of patients' perspectives on technology use as an adjunct to current health care approaches is less well understood. Online health approaches are varied in intention and interaction experience, and elucidation of elements of successful interventions are warranted; the introduction of $m$-health (personal digital assistants and monitoring) and e-health (delivery, surveillance, and health management through telehealth communications) has already substantially altered interactions between consumers and health providers internationally. ${ }^{8-11}$ Telecommunication tools, such as videoconferencing, are also increasingly common in society. ${ }^{8,12-15}$ Asynchronous forms of interaction, such as e-mail, provide another example where patients could pose questions and receive advice from providers without having to wait for formal face-to-face consultation., ${ }^{8,16,17}$ Further investigation is required into the most appropriate approaches in COPD; however, systematic reviews report that telehealth can reduce hospitalization rates and emergency department visits in this population. ${ }^{18,19}$ Quality of life and patient satisfaction were also found to be similar when compared with face-to-face, ${ }^{19}$ and social media and online peer interaction are increasingly used ${ }^{12-14}$ and have been shown to increase social well-being and self-management self-efficacy in individuals with chronic disease. ${ }^{15,20-23}$

A key barrier to online interaction is the view that access is limited in older adults, most burdened with chronic conditions such as COPD, and that older people lack expertise and resources. ${ }^{12,13,23}$ However, a recent report by Pew Internet and American Life Project showed that 64\% of adults older than 65 years use the internet in the United States. ${ }^{24}$ While online health interaction is not as extensive internationally, ${ }^{12}$ it was recently reported in Australia that, 79\% of people older than 65 years accessed the internet over a 12 -month period ${ }^{25}$; this is similar to $86 \%$ of respondents older than 65 years attending a heart and lung clinic, who reported engaging in regular internet use. ${ }^{26}$

Using online or technological health care approaches as an adjunct or replacement of traditional face-to-face health care could bridge geographical and physical barriers experienced by those living with chronic diseases. ${ }^{12-14}$ What is less well understood are patients' perspectives of and interactions with technology-based supports. This study aimed to understand older patients with COPD's perspectives of online approaches to health care.

\section{Materials and Methods DESIGN}

Qualitative study using focus groups and following the consolidated criteria for reporting qualitative research (COR-
EQ) guidelines. ${ }^{27}$ Focus groups were chosen to explore the collective perspectives of complex issues. ${ }^{28-30}$ Interaction around the different ways in which people engage with technology was facilitated through shared reflection and validation of experiences. ${ }^{28-30}$

\section{SAMPLE AND SETTING}

A convenience sample was used of older people with COPD attending pulmonary rehabilitation sessions within a respiratory service at an academic medical center.

Inclusion criteria: Participants were approached by specialist respiratory nurses and physiotherapists and invited to participate if they: were older than 65 years; had a documented diagnosis of COPD; were attending the respiratory service for pulmonary rehabilitation sessions; and able to communicate in English.

Exclusion criteria: Potential participants were excluded if they did not have a diagnosis of COPD or were unable to communicate in English.

\section{PROCEDURE}

Semistructured questions were based on the extant literature and developed in consultation with experts in the field of chronic illness and online health care delivery. ${ }^{19,23,26,31}$ Individuals were facilitated to discuss use of computer and online devices and potential benefits and issues with accessing health information and supports online. To illustrate the variety of online health care approaches that might be available and facilitate discussion, participants were also shown several examples of online sites and resources during the focus groups.

\section{DATA COLLECTION}

Three focus groups, with separate individuals, were undertaken over the study period. Conversations were audio recorded and transcribed verbatim for data analysis. All sessions were facilitated by one investigator who had training and experience in group discussions and with a clinical background in chronic disease management and innovative approaches to health care. The facilitator had no prior or ongoing relationship with the participants beyond the study.

\section{DATA ANALYSIS}

Thematic synthesis of qualitative data was completed in three stages by two or more authors as follows ${ }^{32,33}$ : line-byline free coding of primary data (Stage 1), on individual's perspectives of technology approaches, was kept as close to the text as possible (RTD, SCI). ${ }^{32,34,35}$ Free codes were then organized into descriptive themes (RTD, SCI) (Stage 2) and confirmed through consultation with the author team. ${ }^{32,36}$ 


\section{PERSPECTIVES OF ONLINE HEALTH CARE APPROACHES IN COPD}

Random checking of data extracts for trustworthiness was carried out by a third independent investigator (PMD) and disagreements resolved through discussion. ${ }^{32,36}$ Finally, discussion with an expert panel in the fields of chronic illness and online health care delivery (Stage 3) was used to develop central emergent themes and provide a broader understanding and meaning within the context of patient perspectives of technology health care approaches in COPD. ${ }^{32,36}$ Transparency of the method, the use of independent investigators, and panel discussion were used to promote the validity of findings as well as the rigor and trustworthiness of the synthesis process. ${ }^{28-30,32}$

\section{ETHICAL APPROVAL}

Ethical clearances were approved by the academic and clinical institutions: LHR/13/SVH/5 and 2012-149A.

Three focus groups were undertaken between January and May 2014, with a duration of 55-65 min. Ten patients participated with individuals attending only once. Similar issues were raised across the groups with saturation of perspectives achieved by the final group. All participants had a primary diagnosis of COPD, were older than 65 years, and none required long-term oxygen therapy.

\section{Perspectives of Online-Based Information}

Thematic analysis resulted in five overall themes that described participants' perspectives of online health approaches in people with COPD, including (1) concern over risks in the online environment; (2) multimedia and technology use as part of everyday life; (3) online resources as an opportunity for revision of forgotten knowledge; (5) potential for facilitation of decisionmaking support across geographical and physical barriers; and (4) perceived benefits of online peer support for people with COPD.

\section{CONCERN OVER RISK IN THE ONLINE ENVIRONMENT}

The accuracy, reliability, and variability of internet-based material was a key concern, and in particular, people's ability to find and interpret relevant information: "Google is brilliant, but you could get into, you could get anywhere ... a mish mash of things that aren't helpful"-FG2. Looking up medications online was noted as safer than looking up general health information with participants across the groups already doing this.

Access to too much information was seen as a risk for anxiety, with participants agreeing that while they had the knowledge to interpret what was useful, others may not be as discerning: "I mean, she looks everything up on computer. You can't do that, because if you say 'I've got a tummy ache,' it could be anything. You could be dying of cancer, you could have air, you could have tight pants, who knows!?"-FG3. Others balanced this with the argument that access to information best positioned people to manage their chronic condition, but again raised that direction to credible sources should be a key focus.

Personal information security was only raised in passing by one participant, with reliability and interpretation of information given higher importance to this particular cohort of patients.

\section{MULTIMEDIA AND TECHNOLOGY USE AS PART OF EVERYDAY LIFE}

One key concern for participants was that individuals with COPD may not have access to computers due to general age of the population: "I think it's difficult, for the simple reason the majority of people that have COPD are older ... and even though there are a lot of oldies out there on computers, there are also I think more not"-FG2.

This aside, the majority of this group had access to multimedia devices within the home setting and used these for e-mail, social networking, teleconferencing with friends and grandchildren, and internet browsing. In addition to traditional computers, several participants used smart telephones and tablet devises: in this group, there appeared to be a different relationship between phones and tablets, the former for traditional voice communication and the latter for browsing and e-mailing.

While some participants lacked confidence in technology, relying on children or grandchildren to guide them, others used computers and devices as a part of everyday life and had a strong grasp of technological vocabulary, including "backing up your iPad data to a cloud to avoid losing data in iTunes" and suggestions that to maintain desktop computer memory, "you have to defrag(ment) regularly."

\section{ONLINE RESOURCES AS AN OPPORTUNITY FOR ACCESS TO AND REVISION OF FORGOTTEN KNOWLEDGE}

Access to validated, disease-specific sites was seen as an opportunity to revise prior learning and as "a nice little safety net"-R2. Several participants were still using the paper booklet received during their initial rehabilitation program, and one participant voiced strongly that this was adequate (FG2). However, the currency is questioned given some had received this booklet eight years earlier.

Topics such as "what causes shortness of breath," "how does arm strength reduce shortness of breath," and "depression and COPD" where of particular interest. Participants were interested in online video demonstrations of common strategies and across groups voiced the following: "I had forgotten that," "didn't know that," or "must read that." Loss of knowledge of common strategies, such as "purse lipped breathing" for breathlessness for example, was surprising given their interaction with respiratory physiotherapists through their weekly pulmonary rehabilitation sessions. 


\section{DISLER ET AL.}

Access to information was seen as particularly beneficial to those newly diagnosed with COPD: "In the very beginning, yeah, millions of questions, but you're not sure how to ask them"-FG2. An information source accessed in your own time was seen as positive: "I would have accessed [an online network] to ask that question." Overall, there was interest in accessing information online, particularly for nonurgent queries and tips on how to manage day-to-day challenges. Participants voiced that a site developed in collaboration between health professionals and patients would be ideal.

\section{POTENTIAL FOR FACILITATION OF COLLABORATIVE DECISION-MAKING ACROSS GEOGRAPHICAL AND PHYSICAL BARRIERS}

Traditional face-to-face contact remained the main source of interaction with health care providers; however, across the groups, participants noted that these interactions focused on immediate acute problems with limited opportunity to discuss ongoing and perceived nonurgent challenges. Several participants noted that they delayed perceived nonurgent selfmanagement activities, while waiting for their next specialist appointment: “I mean, look, I haven't even taken that [expectorant prescribed during acute admission] because I want to ask [specialist physician]"-FG2. This aside, several participants did access health professionals through a variety of media, including telephone, fax, and e-mail: "Yeah, I had a couple of questions, because I needed to go on the prednisone ... It was easier to ask by e-mail"-FG1. The level of interaction was determined by the health professional with other participants being restricted to face-to-face 6-monthly or yearly reviews.

Access to timely, collaborative decision-making was seen as highly important, but often limited by wait-times or issues occurring out of hours: "The trouble is if there's a specialist" and "very often when you don't feel well it's night time. It could be one o'clock in the morning"-FG3. Generalist helpline services were overwhelmingly viewed as unhelpful with participants instructed to call emergency services when seeking advice on perceived routine exacerbations. An asynchronous system in which entered symptom and management data could be reviewed by specialized health professionals was viewed with positive interest. A system that could provide tailored self-management information was viewed with equal interest:

While you're in the middle of a bad breathing episode, and you've either got the choice of ringing [emergency services] or sort of getting into a panic, that program, that could calm you down a bit, because when you look at it, you can see the choice of doing certain things to help your breathing-FG2.
Participants recognized that alternate interaction sources would benefit people living outside metropolitan centers: "People in regional areas have very little access to anything. ... They could be sick, but they really need to speak to somebody"-FG3. A negative aspect of online systems, however, was that those lacking technology skills may feel further isolated.

\section{PERCEIVED BENEFITS OF ONLINE PEER SUPPORT FOR PEOPLE WITH COPD}

Online contact with peers was of strong interest. This was particularly important early in disease progression: "In earlier days I wouldn't speak to anyone, well, there was no one that really understood what I was going through." Peers were viewed to bring a different kind of expertise: "Well, it's just like, a doctor can do all the study in the world, but how does he actually know how that person actually feels when you have it? So if you know someone that's been through it or is going through it, or has a chronic condition, this is ideal"-R4 (FG1). Individuals with COPD are commonly socially isolated due to physical debility. Access to peers online was viewed as invaluable: “if you're at home and you're housebound and can't get out, yeah, you need somebody to talk to"-FG1.

Some participants questioned the legitimacy of advice from patients, but others countered that listening to someone who has the same condition was invaluable:

R3: But what he's saying, I mean, he's not trained, he's just a patient, isn't he?

R1: You can relate to his experience, though, it may not be exactly the same, but you could probably relate.

R3: Yeah, when you say that, it makes sense to me too, like that. Ours is a bit different, but I still learn when I learn off your experience-FG2.

Even for these highly engaged patients, many noted that they would access an online social group network if available, and a number were already e-mailing peers in addition to their weekly face-to-face contact.

\section{Discussion}

Participants in this study were older in age and were highly engaged with face-to-face services; however, many were already participating in online health interaction, in a variety of forms, and held overall positive views in this context. The key benefits of online interaction in this study were viewed as increased access to supportive services, decision-making support, and access to peer interaction, reflecting international trends that people older than 65 years and people with 


\section{PERSPECTIVES OF ONLINE HEALTH CARE APPROACHES IN COPD}

chronic disease are increasingly accessing services and support through the online environment. ${ }^{12,20,24,25}$

Individuals with COPD often struggle to access traditional health care services, signaling an important opportunity for online health care approaches. ${ }^{5,8,23}$ Many previous studies have explored patients' perspectives of specific e-health and m-health interventions; however, these have commonly focused on the usability and acceptability of a particular interface or intervention, or to explore perceived benefit on physical functioning or self-management tasks. ${ }^{37}$ The study reported here has sought to explore patients' perspectives around a variety of online approaches and to more broadly explore patients' concerns and thoughts as to how a move to online health interaction and technology-based supports might integrate into management of COPD.

Decision-making support was reported in this study as a vital component of chronic disease management. Timely access to specialized health professionals, when faced with condition fluctuations, was sought after, yet often absent, when needed. ${ }^{38-40}$ Asynchronous online interaction was reported to be of benefit to this cohort, and as noted by others, may facilitate tailored advice without the delays associated with face-to-face consultation wait times. Having timely access to advice created feelings of security in a qualitative study from Norway, which reviewed patient's experience of an mhealth intervention in which COPD health parameters were reviewed by nurses through a web-based portal. ${ }^{41}$ The authors also raised that health professional knowledge of the specific condition was key to confidence in care ${ }^{41}$; similarly, in this study, a lack of confidence was raised when discussing generic telephone helplines with generalist trained staff with limited ability to provide specialist direction.

Obtaining advice on nonurgent issues was identified as a key benefit of online platforms, and in particular, early in disease progression when individuals were overwhelmed with questions, but unsure whom or how to ask. The missed opportunity to ask questions about ongoing issues during health consultations, due to focus on acute issues, has been an ongoing theme throughout previous literature. ${ }^{38-40}$ The subsequent delays in treatment implementation, raised in this study, including changing or starting prescribed medications, are of concern given the known impact of suboptimal disease management on disease progression and symptom burden. ${ }^{31,42}$

Online resources were viewed as a positive opportunity to review information in this study; this reiterated in a Danish study that trialed a web portal within a pulmonary rehabilitation program. While participants in the Danish study reported overall negative experiences with user-friendliness and sought the assurance of face-to-face interaction, they did raise, as seen in this study, that access to online resources allowed them to review information at one's own pace and recap forgotten information. ${ }^{43}$ A key concern for participants, however, was the availability of valid and reliable online information. Misinterpretation of online advice was viewed as dangerous, aligning with similar concerns over health literacy generally ${ }^{44}$ and concerns from a recent American e-health literacy survey of 1,270 patients with COPD, which reported $70 \%$ of patients felt confident in finding health information online, but were less confident in their ability to distinguish the quality of the information. ${ }^{45} \mathrm{~A}$ call for validation of online materials is in line with recent literature, as is the call for health professionals to guide patients to reliable sources, rather than deterring patients from accessing a pervasive and accessible information source. ${ }^{24,26,46}$ Accuracy of patient-measured health parameters was raised as a concern by patients in a Dutch focus group study exploring expectations of patients with COPD, heart failure, and diabetes in a e-health self-management intervention. ${ }^{47}$ However, a phenomenological study of a telemedicine intervention post-hospitalization conversely found that patients felt a positive impact from self-measurement of health data, and that this increased clinical insight and created a mutual clinical language that could facilitate dialogue with the telemedicine nurses. ${ }^{48}$

The introduction of m-health and e-health has substantially altered health interaction and will increasingly provide an important adjunct to traditional forms of health care delivery. ${ }^{9-12,24}$ Moderated sites and direct health provider interfaces may provide the timely and accessible health care interaction to individuals isolated by physical or geographical barriers in particular. Access to reliable information, clear path finding, and computer literacy training are likely to be defining factors in the success or failure of any future online system. ${ }^{49,50}$

\section{LIMITATIONS}

This study was undertaken at a single site with a sample of people who are already highly engaged, although through a face-to-face medium. This does limit generalizability; however, this still does provide important perspectives on online health care approaches and supports in managing this complex condition in this context.

\section{Conclusion}

Participants in this study were older in age, yet held positive views on online health care approaches. They did however raise the need for guidance to valid and reliable online sources. The capacity for online sources to increase access to decision support was of great interest, as was interaction with 
peers. Issues of health care access, increasing health costs, and the need for improved health outcomes drive the search for more effective and sustainable approaches to chronic disease management. Differentiating between online approaches as information strategies and theoretically derived models to promote self-management and facilitate peer support is an important consideration.

\section{Acknowledgment}

The authors thank the patients and staff at the research site for their contribution and involvement in this study.

\section{Disclosure Statement}

No competing financial interests exist.

\section{REFERENCES}

1. Ehteshami-Afshar S, FitzGerald J, Doyle-Waters M, Sadatsafavi M. The global economic burden of asthma and chronic obstructive pulmonary disease. Int J Tuberc Lung Dis 2016;20:11-23.

2. Global Initiative for Chronic Obstructive Lung Disease G. Global strategy for the diagnosis, management, and prevention of COPD. Global Initiative for Chronic Obstructive Lung Disease, Inc., 2018. Available at https://gold copd.org/wp-content/uploads/2017/11/G0LD-2018-v6.0-FINAL-revised-20Nov_WMS.pdf (last accessed October 25, 2018).

3. Fletcher $\mathrm{M}$, Upton J, Taylor-Fishwick J, et al. COPD uncovered: An international survey on the impact of chronic obstructive pulmonary disease [COPD] on a working age population. BMC Public Health 2011;11:612.

4. Spruit MA, Singh SJ, Garvey C, et al. An official American Thoracic Society/ European Respiratory Society statement: Key concepts and advances in pulmonary rehabilitation. Am J Respir Crit Care Med 2013;188:e13-e64.

5. Selzler $A$, Wald J, Sedeno $M$, et al. Telehealth pulmonary rehabilitation: A review of the literature and an example of a nationwide initiative to improve the accessibility of pulmonary rehabilitation. Chron Respir Dis 2017;15:41-47.

6. Zwerink $M$, Brusse-Keizer $M$, van der Valk PD, et al. Self management for patients with chronic obstructive pulmonary disease. Cochrane Database Syst Rev 2014;3:1-165.

7. Mannino DM, Buist AS. Global burden of COPD: Risk factors, prevalence, and future trends. Lancet 2007;370:765-773.

8. Milani RV, Bober RM, Lavie CJ. The role of technology in chronic disease care. Prog Cardiovasc Dis 2016;58:579-583.

9. Kwankam SY. What e-Health can offer. Bull World Health Organ 2004;82: 800-802.

10. World Health Organisation. E-Health. World Health Organization. 2014. Available at www.who.int/trade/glossary/story021/en (last accessed October 25, 2018).

11. Kay M. mHealth: New horizons for health through mobile technologies. World Health Organization, 2011. Available at https://www.who.int/goe/publications/ goe_mhealth_web.pdf (last accessed October 25, 2018).

12. Van Uden-Kraan CF, Drossaert CH, Taal E, Seydel ER, van de Laar MA. Participation in online patient support groups endorses patients' empowerment. Patient Educ Couns 2009;74:61-69.

13. Wicks $P$, Massagli M, Frost J, et al. Sharing health data for better outcomes on PatientsLikeMe. J Med Internet Res 2010;12:e19.

14. Mo PK, Coulson NS. Developing a model for online support group use, empowering processes and psychosocial outcomes for individuals living with HIV/AIDS. Psychol Health 2012;27:445-459.

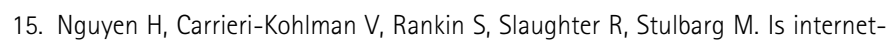
based support for dyspnea self-management in patients with chronic obstructive pulmonary disease possible? Results of a pilot study. Heart Lung 2005;31:51-62.

16. de Jong CC, Ros WJ, Schrijvers G. The effects on health behavior and health outcomes of internet-based asynchronous communication between health providers and patients with a chronic condition: A systematic review. $J$ Med Internet Res 2014;16:e19.

17. Murray E, Burns J, See TS, Lai R, Nazareth I. Interactive health communication applications for people with chronic disease. Cochrane Database Syst Rev 2005;CD004274.

18. McLean S, Nurmatov U, Liu Joseph LY, Pagliari C, Car J, Sheikh A. Telehealthcare for chronic obstructive pulmonary disease. Cochrane Database Syst Rev 2011; CD14007718.

19. Polisena J, Tran K, Cimon K, et al. Home telehealth for chronic obstructive pulmonary disease: A systematic review and meta-analysis. J Telemed Telecare 2010;16:120-127.

20. Chung JE. Social networking in online support groups for health: How online social networking benefits patients. J Health Commun 2014;19:639-659.

21. Nguyen H, Donesky-Cuenco D, Wolpin S, Paul S, Carrieri-KohIman V. Randomized controlled trial of an internet-based versus face-to-face dyspnea self-management program for patients with chronic obstructive pulmonary disease: Pilot study. J Med Internet Res 2008;10:e9.

22. Nguyen H, Gill D, Wolpin S, Steele B, Benditt J. Pilot study of a cell phone-based exercise persistence intervention post-rehabilitation in COPD. Int J Chron Obstruct Pulmon Dis 2009;4:1-13.

23. Nguyen $H Q$, Donesky $D$, Reinke $L F$, et al. Internet-based dyspnea selfmanagement support for patients with chronic obstructive pulmonary disease. $J$ Pain Symptom Manage 2013;46:43-55.

24. Pew Reseach Centre. Internet and Broadband Fact Sheet. 2017. Available at www .pewinternet.org/fact-sheet/internet-broadband (last accessed January 9, 2017).

25. Australian Communications and Media Authority. Digital lives of older Australians. 2016. Available at https://www.acma.gov.au/theACMA/engageblogs/engage-blogs/Research-snapshots/Digital-lives-of-older-Australians (last accessed May 12, 2017).

26. Disler $R$, Inglis $S$, Newton $P$, et al. Patterns of technology use in patients attending a cardiopulmonary outpatient clinic: A self-report survey. J Med Internet Res 2015;4:1-14.

27. Tong $A$, Sainsbury $P$, Craig J. Consolidated criteria for reporting qualitative research (COREQ): A 32-item checklist for interviews and focus groups. Int $J$ Qual Health Care 2007;19:349-357.

28. Creswell JW. Research design: Qualitative, quantitative, and mixed methods approaches, 3rd ed. London: Sage, 2009.

29. Liamputtong P. Focus group methodology: principle and practice. Thousand Oaks, CA: Sage, 2011.

30. Davidson PM, Halcomb E, Gholizadeh L. Focus groups in health research and nursing. In: Liamputtong $P$, ed. Research methods in health: Foundations for evidence-based practice. Australia and New Zealand: Oxford University Press, 2013:54-71.

31. Disler RT, Gallagher RD, Davidson PM. Factors influencing self-management in chronic obstructive pulmonary disease: An integrative review. Int J Nurs Stud 2012;49:230-242.

32. Thomas J, Harden A. Methods for the thematic synthesis of qualitative research in systematic reviews. BMC Med Res Methodo/ 2008;8:45.

33. Barnett-Page $E$, Thomas J. Methods for the synthesis of qualitative research: A critical review. BMC Med Res Methodol 2009;9:59.

34. Massey OT. A proposed model for the analysis and interpretation of focus groups in evaluation research. Eval Program Plan 2011;34:21-28.

35. Liamputtong P, Ezzy D. Qualitative research methods. Melbourne: Wiley Online Library, 2006. 


\section{PERSPECTIVES OF ONLINE HEALTH CARE APPROACHES IN COPD}

36. Walsh $\mathrm{D}$, Downe $\mathrm{S}$. Metasynthesis method for qualitative research: A literature review. J Adv Nur 2005;50:204-211.

37. Early F, Young JS, Robinshaw E, Mi EZ, Mi EZ, Fuld JP. A case series of an off-the-shelf online health resource with integrated nurse coaching to support self-management in COPD. Int J Chron Obstruct Pulmon Dis 2017; 12:2955.

38. Ek K, Ternestedt B-M. Living with chronic obstructive pulmonary disease at the end of life: A phenomenological study. J Adv Nurs 2008;62:470-478.

39. Habraken JM, Pols J, Bindels PJE, Willems DL. The silence of patients with end-stage COPD: A qualitative study. Br J Gen Pract 2008;58:844-849.

40. Gysels M, Higginson IJ. The experience of breathlessness: The social course of chronic obstructive pulmonary disease. J Pain Symptom Manage 2010;39: 555-563.

41. Das A, Bøthun S, Reitan J. A formative evaluation of an eHealth service for patients with COPD. Proceedings of the 4th European Workshop on Practical Aspects of Health Informatics (PAHI 2017). Levanger, Norway: NTNU, 2017.

42. Restrepo RD, Alvarez MT, Wittnebel LD, et al. Medication adherence issues in patients treated for COPD. Int J Chron Obstruct Pulmon Dis 2008;3:371.

43. Cerdan J, Catalan-Matamoros D, Berg SW. Online communication in a rehabilitation setting: Experiences of patients with chronic conditions using a web portal in Denmark. Patient Educ Couns 2017;100:2283-2289.

44. Adams RJ. Improving health outcomes with better patient understanding and education. Risk Manag Healthc Policy 2010;3:61.

45. Stellefson ML, Shuster JJ, Chaney BH, et al. Web-based health information seeking and eHealth literacy among patients living with chronic obstructive pulmonary disease (COPD). Health Commun 2018;33:1410-1424.

46. Adams SA. Revisiting the online health information reliability debate in the wake of "web 2.0": An inter-disciplinary literature and website review. Int J Med Inform 2010;79:391-400.
47. Huygens MW, Vermeulen J, Swinkels IC, Friele RD, Van Schayck OC, De Witte LP. Expectations and needs of patients with a chronic disease toward self-management and eHealth for self-management purposes. BMC Health Serv Res 2016;16:232.

48. Barken TL, Thygesen E, Söderhamn U. Unlocking the limitations: Living with chronic obstructive pulmonary disease and receiving care through telemedicine-A phenomenological study. J Clin Nurs 2018;27:132-142.

49. Wangberg SC, Andreassen HK, Prokosch H-U, Santana SMV, Sørensen T, Chronaki CE. Relations between internet use, socio-economic status (SES), social support and subjective health. Health Promot Int 2008;23:70-77.

50. Wei L. Number matters: The multimodality of internet use as an indicator of the digital inequalities. J Comput Mediat Commun 2012;17:303-318.

Address correspondence to: Rebecca T. Disler, PhD

Department of Rural Health

The University of Melbourne 49 Graham Street Shepparton, VIC 3630 Australia

E-mail: rebecca.disler@unimelb.edu.au

Received: April 10, 2018

Revised: August 4, 2018

Accepted: August 5, 2018

Online Publication Date: November 5, 2018 